\title{
Continuous positive airway pressure
}

\section{treatment for sleep apnoea: compliance increases with time in continuing users}

\author{
M. Sucena, G. Liistro, G. Aubert, D.O. Rodenstein and T. Pieters
}

ABSTRACT: Continuous positive airway pressure (CPAP) remains the best treatment for sleep apnoea syndrome (SAS). In the 1990s, many authors reported on daily compliance, but all of the studies utilised relatively short periods of follow-up that did not exceed a few years.

The mean annual rate of CPAP use in patients with SAS was prospectively recorded. In the current study, the results are presented along with compliance data from patients who started CPAP between 1991 and 1998 and were still using it by the end of 2003. The cohort was chosen in order to obtain $\geqslant 5$ yrs of follow-up for each patient. In total, there were 204 patients. For the whole group, mean \pm SD compliance reached $321 \pm 90$ and $393 \pm 84 \mathrm{~min}$ after 1 and 10 yrs, respectively.

There was no significant change in the first 2 yrs, with a significant increase from the third year onwards. Compliance, or its evolution over time, was not correlated either to the baseline polysomnographical data (except slightly for the CPAP pressure), to the difference of these data before and under CPAP therapy, to the age of retirement or to changes in the marital status.

In conclusion, very long-term compliance with continuous positive airway pressure increases by a mean of $8 \mathrm{~min} \cdot \mathrm{day}^{-1}$ per year of follow-up in patients with sleep apnoea syndrome.

\section{KEYWORDS: Compliance, continuous positive airway pressure, sleep apnoea syndrome}

ompliance with medical treatment is worth considering both in acute and chronic diseases. It is one of the major determinants of treatment failures. In epilepsy, almost half of the patients experience a seizure during follow-up after missing a medication intake [1]. Factors influencing compliance depend on the following: 1) patient personality; 2) interactions between the prescriber and the patient or family; 3) the characteristics of the treatment; 4) its price; and 5) the nature of the disease. For instance, it could be thought that compliance in chronic nonsymptomatic diseases, revealing their deleterious effects years or decades after their diagnosis, such as hypertension or type II diabetes mellitus, requires much more involvement from the patient than compliance in diseases where symptoms are dramatic and unpredictable, such as epilepsy, or compliance among oral contraceptive users. Nevertheless, treatment compliance seems no better in epilepsy than in systemic arterial hypertension [2].

Continuous positive airway pressure (CPAP) has remained the best treatment for sleep apnoea syndrome since its introduction in 1981. Medical staff became rapidly aware of the compliance problems that could be experienced with an external mechanical device. Utilisation rates measured objectively with time counters included in the CPAP devices ranged between 65 and $85 \%$ of the days monitored [3-5]. Most of the studies conducted in the 1990s had relatively short periods of follow-up and were isolated surveys. It has been previously shown that patients used their CPAP for mean \pm SD $5 \pm 1.8 \mathrm{~h} \cdot \mathrm{day}^{-1}$ and that this use was quite stable after treatment onset on two successive follow-up periods of 670 and 390 days on average $(5.2 \pm 1.5$ and $5 \pm 2.3 \mathrm{~h}$, respectively) [5]. In the very long term $(>3 \mathrm{yrs})$, it is not known how patients will behave with respect to their treatment. KRIEGER et al. [6] reported on 728 obstructive sleep apnoea patients who were followed during a mean (range) period of $1,176(27-4,203)$ days. The mean compliance over time was $5.7 \pm 1.8 \mathrm{~h}$, which increased with time. Data were not shown, but one figure indicated a mean use increasing from 5.4 to $6.2 \mathrm{~h}$ during an average follow-up of 4.5 yrs. Since the number of patients was not constant, the fact that the increase was the consequence of the elimination of poor users cannot be discounted.

\section{AFFILIATIONS}

Pneumology Unit, Cliniques Universitaires Saint-Luc, Université Catholique de Louvain, Brussels, Belgium.

CORRESPONDENCE

T. Pieters

Service de Pneumologie

Cliniques universitaires Saint-Luc Université Catholique de Louvain Avenue Hippocrate 10 1200 Brussels

Belgium

Fax: 3227642831

E-mail:

thierry.pieters@clin.ucl.ac.be

Received:

July 282005

Accepted after revision: November 012005
European Respiratory Journal Print ISSN 0903-1936 Online ISSN 1399-3003 
Since 1991, the current authors have prospectively recorded the mean annual rate of CPAP use in patients with obstructive sleep apnoea syndrome. To add new data to the study by KRIEGER et al. [6], the present study was conducted in a fixed population, i.e. those patients who were using CPAP by the end of 2003 and had started treatment between 1991 and 1998.

\section{MATERIAL AND METHODS}

The study cohort consisted of all patients with obstructive sleep apnoea syndrome starting treatment with CPAP between the start of 1991 and the end of 1998, who were still being treated by the end of 2003. The cohort was chosen retrospectively so that all patients would have at least a 5-yr follow-up.

As is the rule in the Belgian social security system, CPAP was only considered for patients with sleep apnoea syndrome complaining of daytime sleepiness and/or snoring and showing, on a full-night diagnostic polysomnography, an apnoea/hypopnoea index (AHI) $\geqslant 20$ and a movement arousal index $(\mathrm{MAI}) \geqslant 30$ per hour of sleep. Some rare patients not fulfilling these criteria also received CPAP therapy, mainly in cases presenting with clear-cut sleepiness and an AHI $\geqslant 20$, but without excessive sleep fragmentation in the diagnostic polysomnography. Details of the methods used were described previously [7]. Movement arousal was defined as the reappearance of an alpha rhythm in the electroencephalogram during a sleep epoch, accompanied by an increase in chin electromyogram activity (both in rapid eye movement (REM) and non-REM sleep) and lasting for $\geqslant 2 \mathrm{~s}$. An apnoea was defined as cessation of airflow for $\geqslant 10 \mathrm{~s}$, whereas a hypopnoea was defined as a $>50 \%$ reduction in airflow for $\geqslant 10 \mathrm{~s}$. The hypopnoea definition required a $>3 \%$ drop in arterial oxygen saturation. Therefore, the desaturation index (DI; number of desaturations $\geqslant 4 \%$ per hour of sleep) was taken as the AHI. Once the diagnosis was established, patients were offered a CPAP trial. Patients were hospitalised for 3-4 nights in order to make them comfortable with treatment. During this period, patients were instructed in the use of CPAP and could try different types of masks, as well as get acquainted with the apparatus. CPAP was only offered to those patients who accepted it and if the polysomnography under CPAP therapy (which directly followed the habituation trial) showed a clear improvement or normalisation of MAI, DI and sleep architecture. The device was delivered to the patient's home by a commercial delegate not belonging to the current authors' unit. This procedure did not change throughout the study period. Once a year, patients were seen in the outpatient's clinic. There was no other reinforcement for treatment, but, in case of trouble or questions, patients could telephone the unit or the technical support service at will. CPAP was free of charge for the patients. Belgium's social security annually reimburses the total cost of the equipment and maintenance, except the humidifier, provided that the mean daily compliance is $>3 \mathrm{~h}$. The maintenance of the CPAP device is also performed yearly at home.

The rate of use reported in the study came from the yearly reading of the built-in time counter by the technical delegate, divided by the number of days separating two readings. In other words, data were collected prospectively. Of this value, $10 \%$ was subtracted to give the daily compliance according to studies comparing running time and effective use $[4,8,9]$. In order to obtain data on the proportion of patients remaining on CPAP therapy for long periods, the patients who quit CPAP and the reasons (intolerance, death or cure) were recorded from 1997 onwards. Therefore, the quit rate and the reasons for quitting were available for the patients of the study groups included during the period of 1996-1998. To gain information on variables that could explain the evolution of compliance with time, and that were not included in the present database, telephone interviews were conducted on all patients who started on CPAP therapy in 1994 and 1996. Questions were asked regarding any change in family status (widowing, divorce or marriage) and the year of retirement for patients having changed their professional status from active workers to retirement. The interviews were performed in July 2004.

The current authors have only systematically recorded subjective sleepiness using the Epworth Sleepiness Scale since 1997. Therefore, such data are not evaluated in this study. The sleep laboratory used had two beds equipped for polysomnographical studies.

\section{Statistical analysis}

First, the differences between the groups (within the year treatment started) were assessed by ANOVA to check the samples' homogeneity for all of the variables studied. The differences within the year of treatment and between successive years of treatment were assessed by ANOVA, followed by post hoc contrasts (least significant difference) as appropriate. The between-group sums of squares were partitioned into trend components to test for a trend of the compliance across the ordered levels of the years of treatment using the contrasts in ANOVA. A regression analysis was used to verify the relationship between compliance and the other variables.

\section{RESULTS}

The mean \pm SD age of the 204 patients included in the present study was $50.6 \pm 10.8$ yrs. A total of 16 females $(8 \%)$ were included. Table 1 shows the characteristics of the population at the time of diagnosis; 10 patients $(5 \%)$ did not fulfil the Belgian criteria for CPAP treatment. All of the patients had a DI of $\geqslant 20$. In this subset, mean (range) MAI was 22 (5-29). From

\begin{tabular}{|c|c|c|}
\hline & Mean & Median \\
\hline Subjects $n$ & 204 & \\
\hline Age yrs & $50.6 \pm 10.8$ & 49.1 (11.6-77.9) \\
\hline BMI $\mathrm{kg} \cdot \mathrm{m}^{-2}$ & $34.9 \pm 7.4$ & $34.2(14.2-64.8)$ \\
\hline DI & $60 \pm 22$ & $60(20-117)$ \\
\hline DI under CPAP & $12 \pm 11$ & $10(0-69)$ \\
\hline MAI & $53 \pm 20$ & $50(5-116)$ \\
\hline MAI under CPAP & $11 \pm 7$ & $9(1-52)$ \\
\hline CPAP pressure $\mathrm{cmH}_{2} \mathrm{O}$ & $9 \pm 2.4$ & $9(5-16)$ \\
\hline \multicolumn{3}{|c|}{$\begin{array}{l}\text { Data are presented as mean } \pm \text { SD and median (range), unless otherwise stated. } \\
\text { BMI: body mass index; DI: desaturation index in the diagnostic polysomno- } \\
\text { graphy; CPAP: continuous positive airway pressure; MAI: movement arousal } \\
\text { index in the diagnostic polysomnography. }\end{array}$} \\
\hline
\end{tabular}




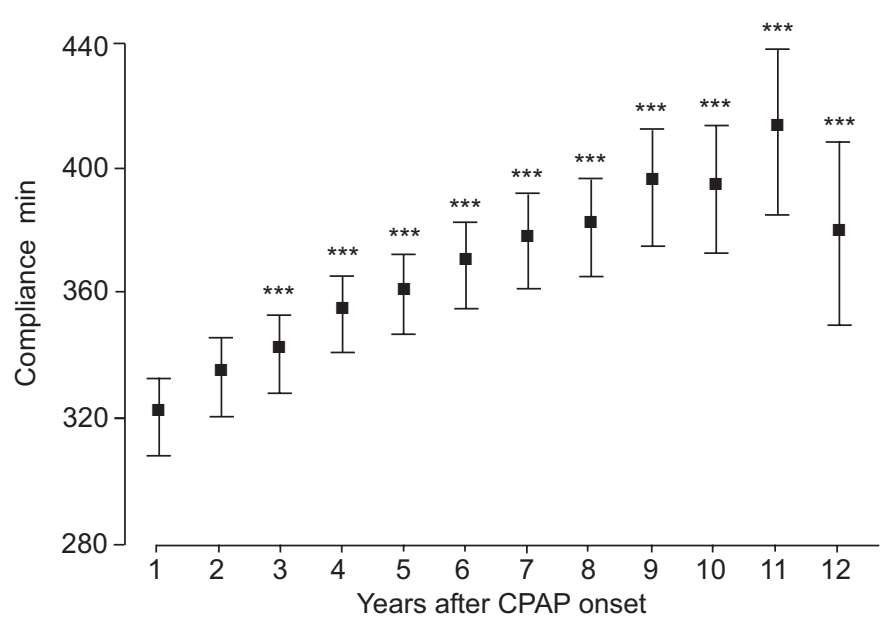

FIGURE 1. Yearly compliance of 204 patients versus time after continuous positive airway pressure (CPAP) onset. Data are presented as mean \pm SD. The number of subjects studied each year was: years $1-5: n=204$ each; year $6: n=176$ year $7: n=134$; year $8: n=112$; year $9: n=88$; year $10: n=67$; year $11: n=53$; and year $12: n=27 .{ }^{* *}: p<0.001$.

1991 to $1998,27,26,14,21,24,22,42$ and 28 patients, respectively, were included each year and were still using CPAP at the end of 2003. There was no significant change in body mass index (BMI) among the different groups of patients throughout the years. The DI was also stable, but the mean \pm SD MAI significantly decreased from 1991 to $1993(68 \pm 22$ and $45 \pm 15$, respectively) and remained stable thereafter $(\mathrm{p}<0.0001)$.

In a univariate analysis, no correlation was found between the compliance during the first year of CPAP treatment and BMI, age, DI, MAI, and difference in DI and MAI between the diagnostic and the therapeutic CPAP polysomnography. Compliance was slightly but significantly correlated with CPAP pressure $(\mathrm{r}=0.145$; 95\% confidence interval $0.3-10.8$; $\mathrm{p}=0.04$ ) so that a higher CPAP pressure was linked to a higher compliance during follow-up. Compliance increased progressively and significantly over time (fig. 1). Mean daily compliance reached $321 \pm 90,360 \pm 92$ and $393 \pm 84$ min after 1, 5 and $10 \mathrm{yrs}$, respectively. The mean slope of compliance increase was $8 \mathrm{~min} \cdot \mathrm{yr}^{-1}$. For the study population as a whole, the difference in compliance with follow-up became significant from the third year of treatment onwards. Figure 2 shows the evolution of compliance in the eight groups of patients starting treatment between 1991 and 1998. Compliance increased in the same way for each group. The evolution was statistically significant except for those patients starting CPAP use in 1998 $(p<0.001$ for patients starting during the period of 1991-1997; $\mathrm{p}=0.001$ for 1993).

The current authors were unable to explain the improvement of the compliance by correlating it to the baseline data (BMI, MAI, DI, CPAP, age, and difference in MAI and DI between the diagnostic polysomnography and the sleep study under (PAP), nor by segregating patients into two groups according to the median value of the variable. The evolution of the compliance was not explained by changes in family or professional status. The answer rate from the telephone interview was $88 \%$. The influence of a change of the CPAP device was also studied. During the study period, 96 patients received a new $\mathrm{CPAP}$ at a given time. They were well matched for MAI, DI, and change in MAI and DI with the patients remaining on the first machine. The two groups showed the same development of mean compliance.

Figure 3 shows the percentage of patients starting CPAP therapy between 1996 and 1998 and followed-up until 2003 who quit treatment. Between 1996 and 1998, 154 patients started CPAP. Of these, $35(22.7 \%)$ abandoned treatment $(14.3$, $3.2,1.9,1.3$ and $1.9 \%$ after $1,2,3,4$ and 5 yrs, respectively). The compliance during the first year of this group of patients was statistically different from compliance of those patients still using CPAP $(179 \pm 120$ versus $321 \pm 102 \mathrm{~min} ; \mathrm{p}<0.001)$. There were also 11 deaths, eight objectively verified cures (DI $\leqslant 20$ and MAI $\leqslant 30$ ), and three withdrawals because the patients were no longer covered by the social security for administrative reasons. Five patients were lost at follow-up because they were transferred to another hospital. Therefore, out of 154 patients starting CPAP, $60 \%$ were still using the devices after 5 yrs.

\section{DISCUSSION}

In a cohort of patients with moderate-to-severe obstructive sleep apnoea surveyed for a long period, with follow-up ranging 5-12 yrs, it was found that compliance to CPAP progressively and statistically increased over time with an average increase of $8 \mathrm{~min} \cdot \mathrm{night}^{-1} \cdot \mathrm{yr}^{-1}$. This is the first time, to the best of the current authors' knowledge, that this could be demonstrated. As the study population remained stable, patients with low use rate who abandoned treatment did not influence the compliance that was obtained. Moreover, the present study demonstrated that this evolution was similar in the eight subgroups of patients according to the year of recruitment into the study. The current authors decided to make this stratification in order to verify that one annual cohort did not influence the results of the whole group. Indeed, all the new patients to whom CPAP was offered and who started treatment during a given year showed the same evolution and, in almost all cases, the increase of use with time was extremely significant from the statistical point of view. The difference in compliance reached significance from the third year onwards. These results confirm previous data showing a stable compliance in a small subgroup of 36 patients followed during 3 yrs [5]. According to the analysis of the cohort included between 1996 and 1998,60\% of these patients used their devices for $>5$ yrs. This cohort is representative of the whole population.

It is not known whether such data are applicable to other settings. In fact, there are no such data in the literature. The present study is a first step and others are necessary to allow comparisons.

Compliance to CPAP has been shown to be weakly correlated to baseline disease severity and better to subjectively felt benefits [10]. Most of the previous studies were surveys of short duration (months or a few years). It has been shown previously that compliance after a mean follow-up of 784 days was correlated to baseline MAI $(r=0.226 ; p<0.05)$ [5]. In the present study, none of the considered baseline variables 


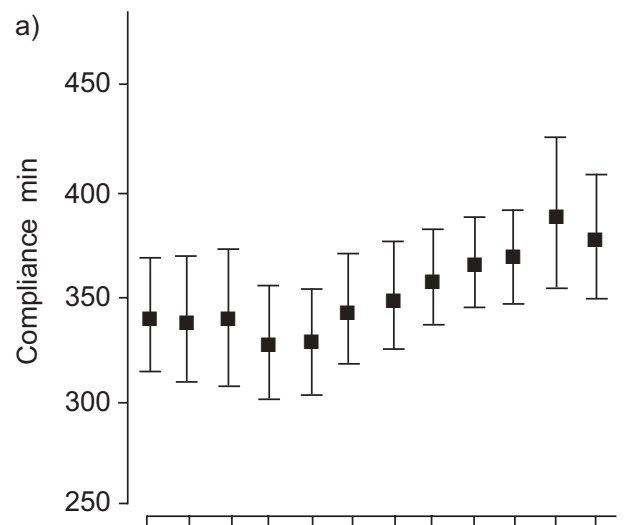

b)

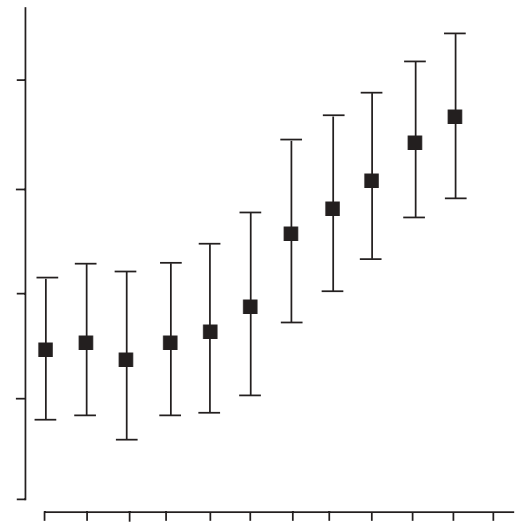

d)
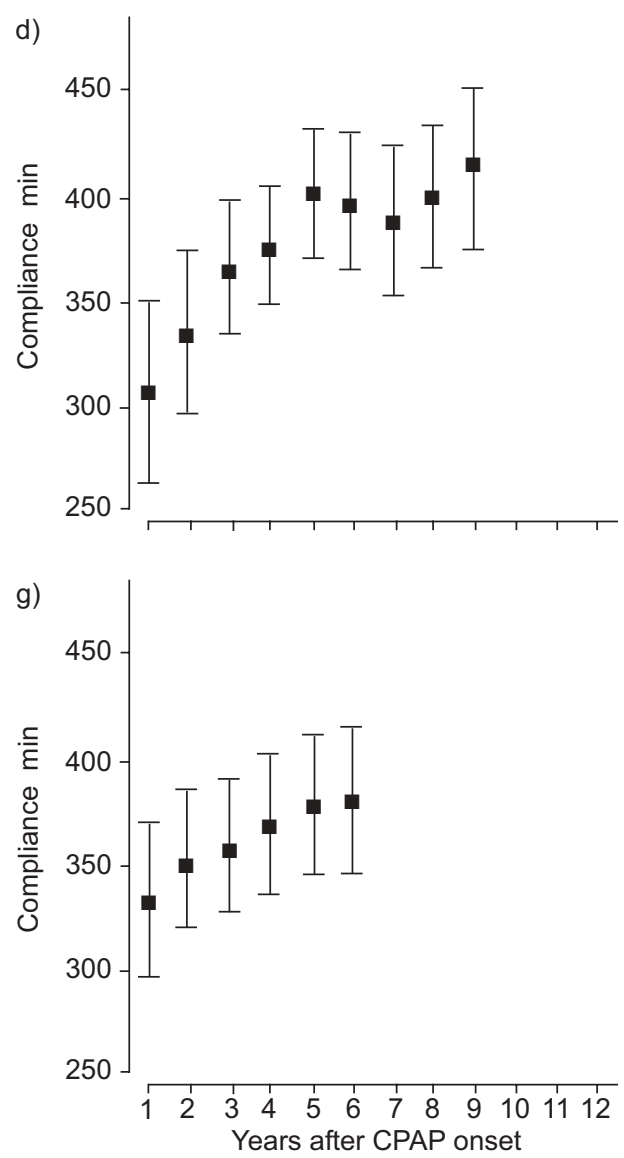

e)

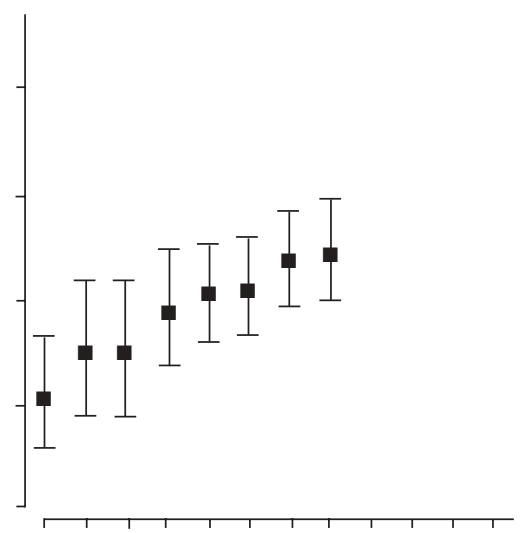

h)

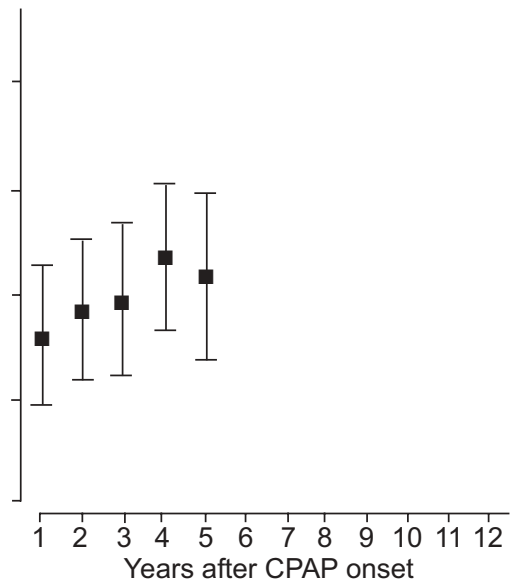

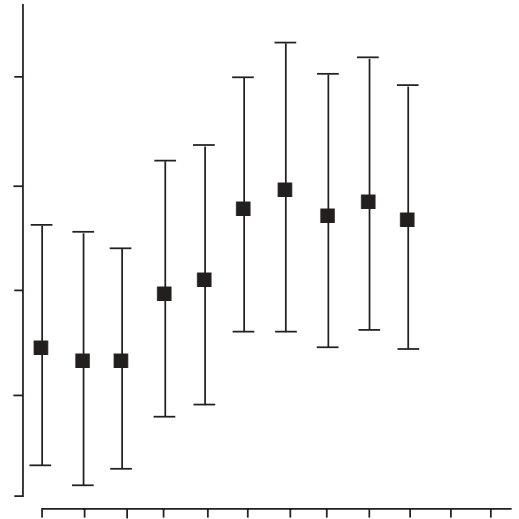

f)

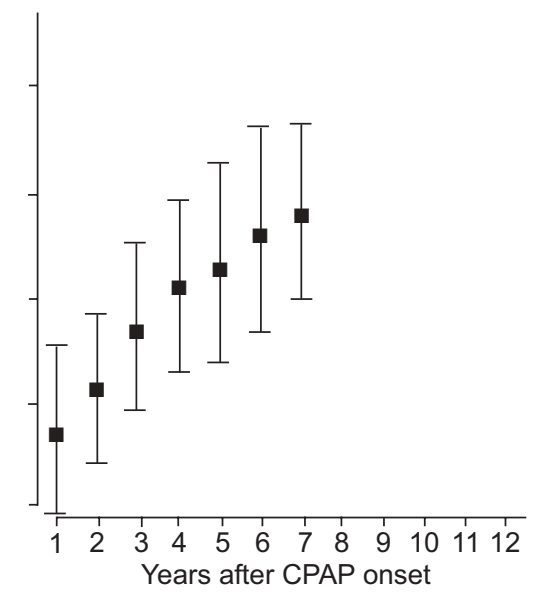

FIGURE 2. Yearly compliance of each subgroup of patients according to the year of inclusion in the study after continuous positive airway pressure (CPAP) onset from 1991-1998 (a-h, respectively). Data are presented as mean $\pm \mathrm{SD}$. a, $b, d-g: p<0.001 ; c: p=0.001 ; h$ : nonsignificant.

(except CPAP pressure) correlated to compliance or to its evolution with time. Recently, LEWIS et al. [11] showed that compliance at 1 month was not correlated to baseline anxiety, depression scores, smoking or alcohol status, concomitant pulmonary disease, or to the presence of a moustache or beard. Conversely, patients experiencing no initial tolerance problems to CPAP and living with a partner were better compliers [11]. To explain the increase in compliance over time, an eventual change in the family status was also explored. Since this information was not available in the current prospective data set, it was decided to perform telephone interviews of all the patients in two selected subgroups where the increase in compliance was without doubt (1994 and 1996). None of the patients had been widowed, divorced or married during the follow-up period. SiN et al. [12] showed that compliance at 6 months was significantly correlated with female sex, reduction in a sleepiness score and increasing age. The mean compliance was $5.8 \mathrm{~h}$ at the end of the study period and was comparable to previous studies. In the study by SIN et al. [12], a 10-yr increment in age was associated with a mean increase of 15-min CPAP use. In the present longitudinal study, it has been shown that the 10-yr increment in CPAP use in a fixed group of patients averaged $80 \mathrm{~min}$. If patients used their CPAP more, this could be explained by more time asleep. Retirement is the best way to get more sleep hours in active patients. In the same telephone survey, the current authors looked at this variable, but were not able to demonstrate, on an individual basis, a link of the increase in CPAP use with the cessation of the occupational career. The eventual influence of a change in the CPAP device was studied, but in the entire study group. 


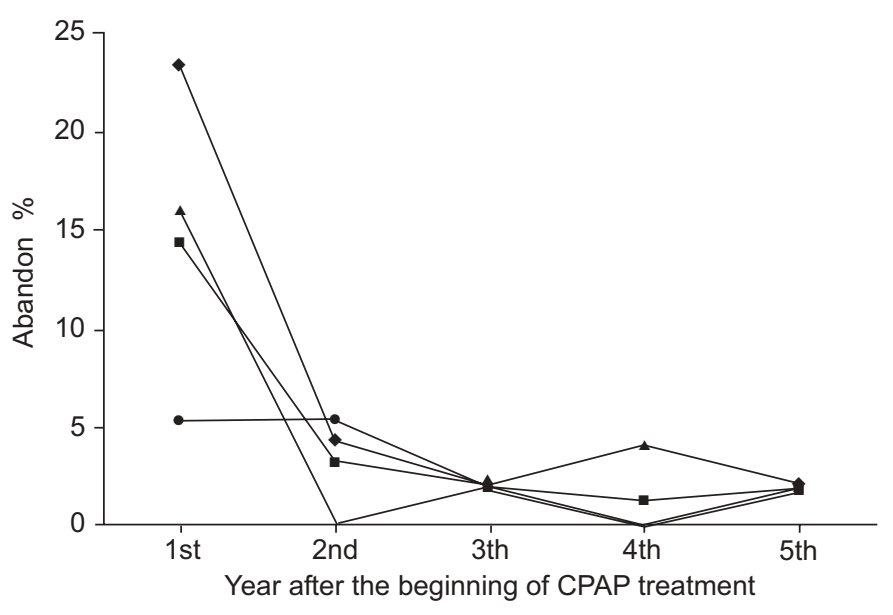

FIGURE 3. Rate of abandoning continuous positive airway pressure (CPAP) in patients starting CPAP between 1996 and 1998. : 1996; •: 1997; $\mathbf{\Delta}$ : 1998; mean.

The most modern devices are smaller, lighter and less noisy and could, therefore, facilitate adherence. Patients received a new device when the old one became noisy or had an electronic failure. When comparing those patients who received a new device with those still using the original CPAP at the end of the study period, no difference in mean compliance or in its evolution was found. Technological advances in mask interfaces would also be expected to improve compliance. Indeed, in the present study population, masks were replaced almost every 1-2 yrs at the discretion of the patient or the technical delegate who performed the yearly maintenance check. Unfortunately, this item was not prospectively recorded in the study database.

Noncompliance to CPAP is understandable. Wearing a CPAP device is all but convenient. Air leaks, nasal blockage or discharge, soreness, redness and ulceration of the skin, claustrophobia or aerophagy, and noise are the most common side-effects. A corrective measure can be offered for the vast majority of side-effects. The only one for which no solution exists is the sensation of claustrophobia and invasiveness induced by the mask, head girdle and air pressure. Many patients quit or even do not start treatment for this reason. The acceptance rate reached $76 \%$ in the current authors' previous study [5]. It was not determined in the present one. The quit rate once treatment was started was $11 \%$ in a previous study, in the range found by others. Parallel to this study, the present authors re-examined this point and found a quit rate of $22.7 \%$ after 5 yrs. It was more important during the first year $(14.3 \%)$ and was plateauing to a few per cent from the second year. Other patients may try to find a compromise to the discomfort of CPAP. By using it at a minimum level, it was found to be sufficient to counteract the deleterious effects of sleep apnoea syndrome. This level sometimes lies below the minimal level imposed by the rules of the Belgian social security. In Belgium for instance, this level is set arbitrarily at $3 \mathrm{~h} \cdot$ night $^{-1}$. In the present study, 11 patients used their CPAP $<3 \mathrm{~h}$ during the first year. All but one patient became "normal" compliers at the end of the study period, contrary to the current authors' previous study, with a follow-up of 1 yr after an initial survey of $2 \mathrm{yrs}$, where poor compliers $\left(<3 \mathrm{~h} \cdot \mathrm{day}^{-1}\right)$ remained the same at $1 \mathrm{yr}$ after the initial survey.

The present data confirm that early low compliance is a feature of patients quitting treatment in the first year of use. Moreover, most patients who quit do so in the first year of treatment. This suggests that reinforcing interventions may be important early after the introduction of CPAP therapy, but that they are unnecessary thereafter. Moreover MCARDLE et al. [13] studied 1,103 patients with a median follow-up of 22 months and found that compliance at 3 months was strongly predictive of long-term use. If it is considered that, according to previous studies [4, 13], 4.5-24\% of patients needing CPAP refuse the therapy and that 20 [13] to $22.7 \%$ (current data) of the patients who start it will quit CPAP within 5 yrs, then almost $60 \%$ of all patients accept and comply with therapy on very long-term follow-up [5, 13]. This is similar to what is found in most other chronic diseases with the notable difference that compliance tends to decrease with time receiving therapy in these diseases [14].

In conclusion, it was found that patients started on continuous positive airway pressure, provided they do not quit in the first year of therapy, increase the therapeutic compliance by an average of $8 \mathrm{~min} \cdot \mathrm{day}^{-1}$ each year of continuing therapy on very long-term follow-up.

\section{REFERENCES}

1 Cramer J, Glassman M, Rienzi V. The relationship between poor compliance and seizures. Epilepsy Behav 2002; 3: 338-342.

2 Ross S, Walker A, Macleod MJ. Patient compliance in hypertension: role of illness perceptions and treatment beliefs. J Hum Hypertens 2004; 18: 607-613.

3 Krieger J. Long-term compliance with nasal continuous positive airway pressure (CPAP) in obstructive sleep apnea patients and nonapneic snorers. Sleep 1992; 15: Suppl. 6, S42-S46.

4 Kribbs N, Pack A, Kline L, et al. Objective measurements of patterns of nasal CPAP use by patients with obstructive sleep apnea. Am Rev Respir Dis 1993; 147: 887-895.

5 Pieters T, Collard P, Aubert G, et al. Acceptance and longterm compliance with CPAP in patients with obstructive sleep apnoea syndrome. Eur Respir J 1996; 9: 939-944.

6 Krieger J, Kurtz D, Petiau C, et al. Long-term compliance with CPAP in obstructive sleep apnea patients and in snorers. Sleep 1996; 19: Suppl. 9, S136-S143.

7 Rodenstein DO, D'Odemont JP, Pieters T, AubertTulkens G. Diurnal and nocturnal diuresis and natriuresis in obstructive sleep apnea. Am Rev Respir Dis 1992; 145: 1367-1371.

8 Engleman H, Martin S, Douglas N. Compliance with CPAP therapy in patients with the sleep apnoea/hypopnoea syndrome. Thorax 1994; 49: 263-266.

9 Reeves-Hoche M, Meck R, Zwillich C. Nasal CPAP: an objective evaluation of patient compliance. Am J Respir Crit Care Med 1994; 149: 149-154.

10 Engleman $\mathrm{H}$, Wild M. Improving CPAP use by patients with the sleep apnoea/hypopoea syndrome (SAHS). Sleep Med Rev 2003; 7: 81-99.

11 Lewis K, Seale L, Bartle I, et al. Early predictors of CPAP use for treatment of obstructive sleep apnea. Sleep 2004; 27: 134-138. 
12 Sin D, Mayers I, Man G, Pawluck L. Long-term compliance rates to continuous positive airway pressure in obstructive sleep apnea. A population-based study. Chest 2002; 121: 430-435.

13 McArdle N, Devereux G, Heidarnejad H, Engleman HM, Mackay TW, Douglas NJ. Long-term use of CPAP therapy for sleep apnea/hypopnea syndrome. Am J Respir Crit Care Med 1999; 159: 1108-1114.

14 de Klerck E, ven der Heijde D, Landewe $R$, van der Tempel H, Urquhart J, van der Linden S. Patient compliance in rheumatoid arthritis, polymyalgia rheumatica, and gout. J Rheumatol 2003; 30: 44-54. 